\title{
Probabilistic Modeling of Roughness Effects Caused by Bio- Colonization on Hydrodynamic Coefficients: A Sensitivity Study for Jacket-Platforms in Gulf of Guinea
}

\author{
AMERYOUN Hamed and SCHOEFS Franck \\ LUNAM Université, Université de Nantes-Ecole Centrale Nantes, GeM, Institute for Research in Civil \\ and Mechanical Engineering/Sea and Littoral Research Institute, CNRS UMR 6183/FR 3473, Nantes, \\ France- Corresponding author: Franck.Schoefs@univ-nantes.fr \\ France Ph.: +33 (0) 2.51.12.55.22 \\ hamed.ameryoun@univ-nantes.fr \\ Franck.Schoefs@univ-nantes.fr
}

\begin{abstract}
Nowadays, challenge for requalification of existing offshore platforms through the reassessment process leads to consider the importance of updating new information (e.g. environmental data, new regulations, etc.). Regarding to this information and depending on offshore fields, data of marine growth colonization is shown to have a dominant effect. This is a real challenge in the Gulf of Guinea that this study focuses on. Marine growth is known to cause adverse effects on the performance of offshore structures. Its presence can change the roughness and the diameter of structural members and hence change the level of hydrodynamic coefficients. Moreover, modifying the added mass can change the natural period and hence dynamic responses of structures. Even platforms with the best protection schemes against marine organisms will after few weeks at least few months start to be covered by various types of marine growth. Generally, it was also recognized that the most important source of loading exerted on offshore structures comes from hydrodynamic actions which are influenced by hydrodynamic coefficient values. The colonization process is very complex and results are in a large diversity of marine growth type (animal, vegetal- hard, soft) and species. This study therefore proposes a stochastic modeling of marine growth and the roughness of hard species based on Response Surface Methodology. A geometrical description of $n^{\text {th }}$ order of Stokes model, formed by a random linear combination of deterministic vectors is employed. Finally, the complexity level of roughness modeling is analyzed and the results are discussed.
\end{abstract}

\section{INTRODUCTION}

Reassessment of offshore structures is became one of the major operational challenges for industries. It results to update the design hypothesis of these structures that deals with topics such as structural behavior, environmental conditions and the structural integrity. The present paper takes place in the mentioned challenge for the reanalysis and to extend the lifetime of existing jackets where the uncertainties have to be controlled based on the analysis of inspection reports. Previous studies are generally focused on two types of questions: crack detection and measurement or marine growth assessments. This paper aims to analyze the effect of the roughness caused by the latter on hydrodynamic coefficients based on the response surface method.

Indeed, biocolonization on offshore structures is of major importance as it can increase the screen effect and modify the hydrodynamic flow near the structure. This paper firstly reviews the general effects of the biofouling on jackets and lies the overloading and increasing of the roughness due to the biocolonization.

The response surfaces of the $\mathrm{R}_{\mathrm{e}}$ and $\mathrm{Kc}$ in presence of the marine growth are proposed. A discussion on uncertainty and sensitivity studies is carried out. Finally the study will be finished with the roughness modeling hypothesis and the discussion on the results after Monte-Carlo simulations of drag forces.

\section{GENERAL EFFECTS OF MARINE GROWTH ON JACKET PLATFORMS}

When a structure is immersed in the seawater, it is rapidly covered by the unavoidable marine organisms. Its nature and growth are complex phenomena and a lot remains misunderstood. The structures which are partially built afloat or transported to the exploitation site may have the larvae of marine organisms attached before they arrived at their installation locations [1]. Then geographical location, distance from the shore, soil nature, the water depth, building material including coatings and the type of structure, waves, water 
currents, nutrients availability, physicochemical parameters of seawater, presence of protection systems, human activities, and the date of platform installation in relation with the date of release of spores and larvae will affect the bio-fouling nature and growth rate [2]. The superposition and competition of these mechanisms make the marine growth modeling still a challenge for actual research. Recent measurements [3] demonstrate that marine growth thickness may be considerable (superior to 150 $\mathrm{mm}$ ) depending on geographical site locations. They may have a significant impact on logistics and cost of structural inspections and operational maintenance programs.

The marine growth can damage underlying hard substrata through some mechanisms such as mechanical degradation, chemical degradation and bacterial activities associated with specific micro-environments that are created by marine growth. They can be caused by recurring cleaning of structures, the action of storms, bacterial activities associated with specific microenvironments created by marine organisms or the presence of cathodic protection devices that are typically used to protect metal platforms against the corrosion $[4,5]$. The quantification of damage caused is generally unknown and it is not revealed in the existing investigations [6].

\section{Overloading from marine growth modeling and measure}

It is instructive to firstly mention the relevant contributions in the literature, which can be categorized into two groups: (i) hydrodynamic modeling from experiments and (ii) marine growth physical modeling from in-situ measurements.

Concerning the first group, many research studies have been done on the basis of sensitivity studies of overloading due to the nature and thickness of marine growth and hydrodynamic structure of flows. As the techniques of flow velocity and acceleration measurements are particularly in-depth, studies are mostly recent in this field. In fact, the equipments in water tunnels or large basins are generally costly considering the fact that large-scale experiments must be considered for similitude conservation. Some of them are usefully reviewed and detailed by Sarpkaya [30]. The pioneer works of Sarpkaya [8, 9] concern fixed roughened cylinders placed in a sinusoidal oscillating flow. Others consider roughened cylinders subjected to sinusoidal oscillation in a large basin with the intention of promoting two-dimensional flow [5]. After this period, another way of experimentation concerns the carriage technique i.e. forced sinusoidal oscillations of a cylinder in a basin [10, 1, 11]. The relative Roughness i.e. Roughness $k$ divided by diameter $D$ of clean cylinder tested varies from $1 / 240$ to $1 / 50$. These works contributed largely to a better understanding of relations between the nature and thickness of fouling and relative changes of loads. Systematic measurements of Reynolds, Keulegan-Carpenter and Sarpkaya numbers allow us to assess precisely the hydrodynamic conditions of the tests. In spite of 'controlled laboratory conditions' significant differences in drag, inertia and lift coefficients data are found especially for Keulegan-Carpenter exceeded 8 with no predominant reason [7] while the effects of three-dimensional vortex instability may affect them. A specific work carried out by Theophanatos pointed out that the one-parameter characterization of relative roughness $(k / D)$ is not suitable to embrace all the complexity of fluid-structure interaction and to quantify loading [12]. In fact the shape of roughness, effects of soft seaweeds, limitation of experimental scales and percentage of coverage have been shown to have a great influence on the results and should be taken into account. Note that in some sites like in the Gulf of Guinea for instance, waves and currents coexist with a similar contribution to loading and special effects of their combination should be studied.

Note that another effect of marine growth is the overweight: it can be of the same order of magnitude as the weight of the clean structure itself and hence it can change the demand on foundations.

\section{Effect of roughness increasing on hydrodynamic coefficients}

Marine growth can result in increasing surface roughness and hence changes in both of the drag and inertia coefficients being linked to the hydrodynamic forces across Morison equation [13]. Their variations induced by the presence of marine growth, influence the hydrodynamic forces in nonlinear way. The percentage of coverage also affects the hydrodynamic coefficients and hence the forces, especially for percentage smaller than 25\% [12]. Figure 1, illustrates the evolution of drag coefficient as a function of surface coverage. Moreover, increasing of the marine growth density could lead to decrease in the natural frequencies of the structure. Indeed, marine growth density has a strong influence on the torsion and third force-aft and side-to-side natural frequencies of the support structure [14].

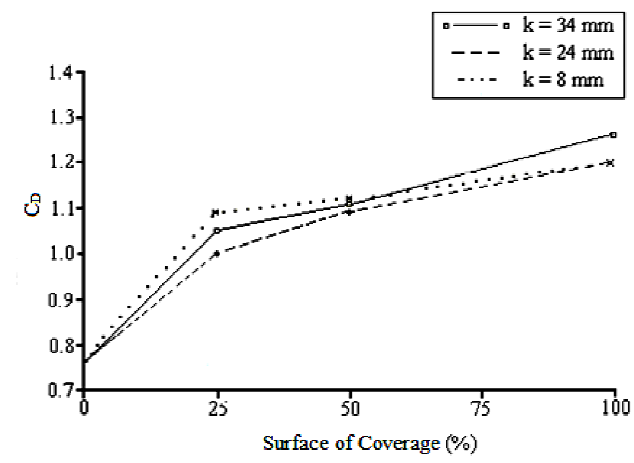

Figure 1. Evolution of the drag coefficient as a function of surface coverage [12].

Sarpkaya in [9] demonstrates by experimental trials that roughness variations can generate variations of drag and inertia coefficients of the Morison equation [8]. The results show that the drag coefficient is twice as great for rough cylinders in comparison to smooth cylinders. It also grows with the relative roughness $k / D$. Figure 2, presents the variations of hydrodynamic coefficients versus Reynolds number for different relative roughnesses, $k / D$, here $k$ is the average size of the roughness of the colonization of fouling and $D$ the nominal diameter of the cylinder, ranging from $1 / 800$ to $1 / 50$. 


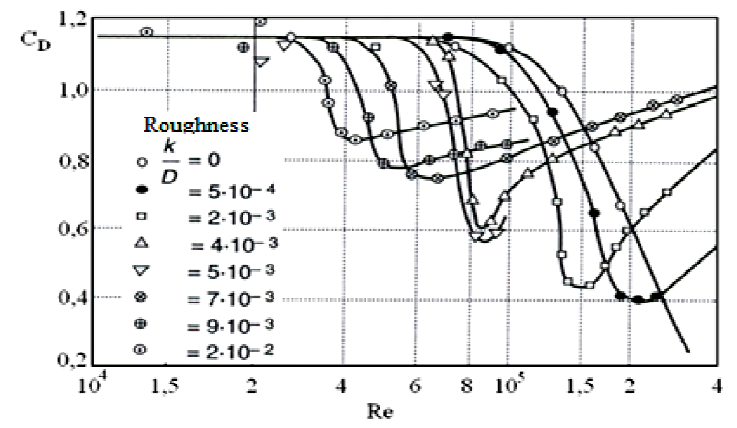

Fig. 2. Effect of roughness on the drag coefficient [15].

\section{STOCHASTIC PROCESSES OF MARINE GROWTH MODELING.}

Considering the effect of biofouling on the hydrodynamic coefficients in Morison equation [16], some researchers have proposed a model for the drag coefficient as a linear regression function of the thickness and roughness [1]. However, their results showed a very high dispersion and they concluded that additional examinations were required. There will be always uncertainties due to errors in the measurement of physical parameters of bio-fouling (i.e. the thickness, length and percentage cover). Although the mentioned study provides further guidance to advocate the process of bio-fouling colonization from the perspective of modeling, like probabilistic modeling, but the stochastic process of the biocolonization is generally unknown. It depends heavily on short-term phenomena such as severe storms that can clean some structures and electro-chemical effects of cathodic protection [16]. Local climate changes can also affect the life or growth of some species. That is why coefficients $C_{D}$ and $C_{m}$ should be considered both as hydrodynamic coefficients and parameters for error modeling [17].

Considering the mechanical behavior of offshore structures subjected to harsh environmental conditions, the reliability analysis of structures offers a set of robust tools and methods. It reveals the importance of uncertainty and sensitivity analysis especially in view of characterizing the stochastic structure of the loads which act on the offshore structures $[17,18]$. Firstly, the innate randomness of waves and the modeling uncertainty of fluid-structure interaction in their relative contribution to the response (i.e. the loads on the structure) should be considered. Particularly, there is a need to account for the natural organism clusters such as marine growth colonization (e.g. kelps, mussel beds, etc.) in contrast to the probabilistic modeling. In fact, this settlement of the structures includes the major uncertainty source in terms of diameter increasing and hydrodynamic coefficient levels. Its corresponding uncertainty includes several factors (e.g. seasons, depth, etc.) during the life-time of the structures and is greater at the design stage when no inspection reports are available. As for steel fixed-type offshore structures like Jacket or Jack-up platforms, the hydrodynamic overloading caused by marine growth is the major factor in terms of percentage of coverage on each component and consequently on their summation for the external global loading assessment. Effects on cylinders with small diameters compared to wave lengths are considered in this study.

\section{Response surfaces of $\mathrm{Re}$ and $\mathrm{KC}$ in case of marine growth presence}

From Morison equation and the established response surface of the kinematic filed, a response surface of the distributed forces can be deduced in the form of:

$$
\begin{gathered}
\operatorname{Re}=\frac{\max _{t \in[0 ; T / 2]}\left(\left\|a(t)_{(n)} \sin \left(\underline{\alpha_{1}}\right)-b(t)_{(n)} \cos \left(\underline{\alpha_{1}}\right)\right\|\right) D_{c}}{v} \times \theta_{m g}(1) \\
K c=\frac{\max _{t \in[0 ; T / 2]}\left(\left\|a(t)_{(n)} \sin \left(\underline{\alpha_{1}}\right)-b(t)_{(n)} \cos \left(\underline{\alpha_{1}}\right)\right\|\right) T^{(2)}}{D_{c} \times \theta_{m g}}
\end{gathered}
$$

Where $\theta_{m g}$, is a multiplier stochastic process indexed in Time-Space and is dependent on the marine growth thickness process. It allows taking into account the increment diameter of structural elements and can be deduced from mean thickness of the marine growth by the relation below:

$$
\theta_{m g}=1+\frac{2 \times t \bar{h}(Z, t)}{D_{c}}
$$

Where, $D_{c}$ is the nominal diameter of the clean element.

The equivalent diameter of the structural elements can be given by:

$$
D=\theta_{m g}(Z, t) \times D_{c}
$$

The hydraulic coefficients $C_{X}, C_{D}, C_{X}^{\prime}$ and $C_{M}$ are dependent on the hydraulic parameters $\left(R_{e}, K_{C}\right)$, the wave height and the associated period and thickness of marine growth, the parameter of $\theta_{m g}$ that affects the hydrodynamic coefficients through the relationships between the hydraulic parameters and the diameter of the elements. So the hydraulic parameters of the horizontal component of the speed in the presence of marine growth can be given by:

$$
\begin{gathered}
\operatorname{Re}=\frac{\max _{t \in[0 ; T / 2]}\left(\left\|a(t)_{(n)} \sin \left(\underline{\alpha_{1}}\right)-b(t)_{(n)} \cos \left(\underline{\alpha_{1}}\right)\right\|\right) D_{c}}{v} \times \theta_{m g}(5) \\
K c=\frac{\max _{t \in[0 ; T / 2]}\left(\left\|a(t)_{(n)} \sin \left(\underline{\alpha_{1}}\right)-b(t)_{(n)} \cos \left(\underline{\alpha_{1}}\right)\right\|\right)^{T}}{D_{c} \times \theta_{m g}}(6)
\end{gathered}
$$

The hydrodynamic coefficients, $C_{D}, C_{X}, C_{M}$ and $C_{X}^{\prime}$, are a function of hydraulic parameters (numerical interpolation) and then the coefficient of $\theta_{m g}$, so these are stochastic fields indexed in $Z$ and t. $a, b, c$ and $d$, are stochastic fields dependent on $H$ and $T$, are indexed in $x$ and $z$ [4].

Here, only $K_{C}$ is needed because the flow regime is postcritical $\left(R_{e}>10^{6}\right)$. Note that this bound is questionable because it is itself dependent of marine growth roughness. For simplicity here, this bound is admitted as correct. 


\section{DISCUSSION ON UNCERTAINTY AND SENSITIVITY STUDIES}

The response surface employed in this study is built around the transfer of the energy of the wave kinematics fields due to Morison equation. It leads to the discussion of uncertainty modeling in the model of kinematics field of water particles and the sensitivity of the transfer of its energy to complexity level. Sensitivity studies usually concern:

I. Complexity of the environmental modeling: wave, wave + current, and finally wave + current + wind.

II. Complexity of the kinematics field modeling: order of Navier-Stokes model and accounting for current or not and role of modeling of wave-current conditions.

III. Modeling of hydrodynamic coefficient in the Morison equation.

IV. Modeling of marine growth: Roughness, Thickness and percentage of coverage.

Several studies already discussed some items:

(II.) in [17, 19].

(III.) in Schoefs and Boukinda [18] and Schoefs and Boukinda [20].

Due to the specificity of the Gulf of Guinea, this study focuses on (IV.) modeling of effect of marine growth with time. Questions such as sensitivity of percentage of coverage and wave-current distributions are out of the focus of this paper.

Sensitivity studies are conducted at three levels:

Bio-fouling conditions for which three steps are considered: smooth tube (installation time or after cleaning), colonized tube in 10 years and colonized tube in 33 years. The surface relative roughness $(k / D)$, for three conditions is presented in Figure 3. This figure corresponds to 0.021 for $t=10$ and 0.046 for $t=33$ years. According to the API curve, corresponding $\mathrm{C}_{\mathrm{DS}}$ are given to 0.6 for smooth tubes, 1.088 for tubes colonized during 10 years and 1.101 for tubes colonized during 33 years. Because of direct exploitation of API curves, for rough cylinders the value of 1.2 is noted as is recommended.

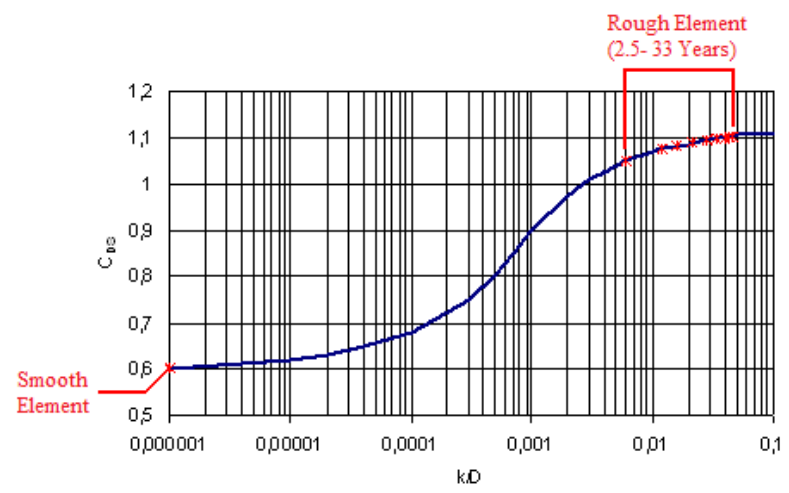

Figure 3. Determination of CDS as a function of surface roughness and time at $\mathrm{t}=0,10$ and 33 years [21].

The level of complexity can be generated by two levels of study to calculate the distributed forces, once the choice of the order of Stokes wave made:

- The ocean-meteorological conditions with the influence of inclusion of offshore wind sea on the statistics of hydraulic coefficients, hydrodynamics and forces.

- $\quad$ The probabilistic modeling of $C_{D}$.

For this last point and to avoid of multiply the case study, only vertical elements under the wave crest are analyzed, this implies high horizontal speeds and accelerations that generate very small forces: the inertia forces are very low.

For these different studies, the hydraulic parameters indicate whether the flow regime is post-critical and the range of the transfer function of $K_{C}$ to $C_{D}$ which are used. A comparison with the currently used deterministic approach is proposed.

It should be able to offer a sampling of the thickness of the marine growth, $t h$, and thus $\theta_{m g}$.

The parameters of first variable $\left(\theta_{m g}\right)$ can be assumed as a random variable independent of the sea-state parameters $(\mathrm{H}$ and $\mathrm{T})$ with a symmetrical triangular distribution of $\mu \pm 2 \sigma$. It is clear that there are some uncertainties in the prediction of forces applied to the marine works that can be taken into account in the magnitudes of fluid-structure interactions (coefficients of Drag and Inertia) and on the amount or distribution of marine biofouling.

\section{Effect of marine growth roughness}

Corals are the dominant species in this study. From the photos of colonization taken by ROV on a platform in the study region, 30 years after its installation, the roughness was visually estimated to $60 \mathrm{~mm}$ as it is presented in Figure 4.

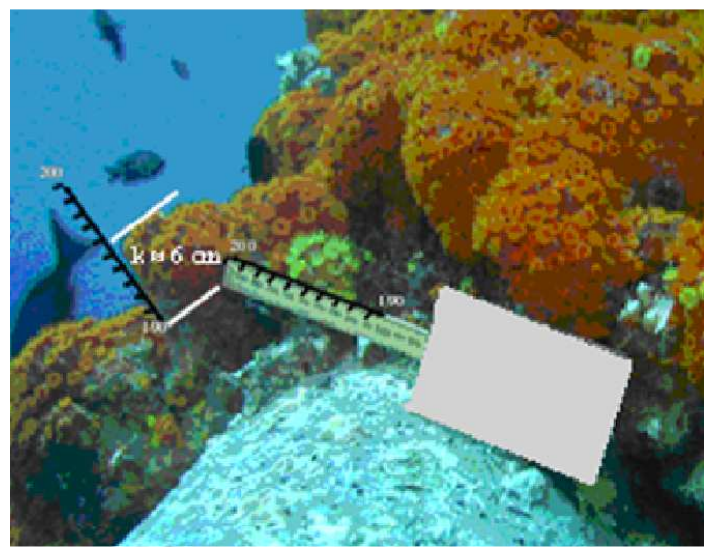

Figure 4. Determination of the roughness of corals [6].

A linear evolution of roughness $(k)$ has been assumed since the installation phase of the platform up to 30 years. To stay in the most possible realistic conditions, we hypothesized that after 30 years, roughness will be constant as it is presented in Figure 5. Indeed the roughness cannot increase indefinitely with time. The conditions of marine growth were studied at $\mathrm{t}=0$, $2.5,5,7.5,10,15,20,25,30$ and 33 years. For a rough element, the roughness of surface varies between 0.006 and 0.046 which corresponds to a range of variation of $C_{D S}$ from 1.05 to 1.102 (see Figure 3). 


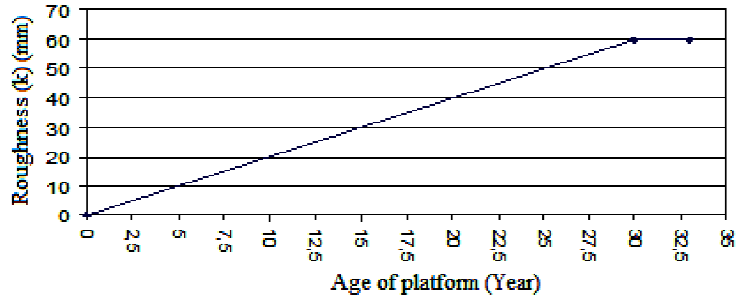

Figure 5. Evolution of roughness with time.

The biofouling community can change its nature and the colonization process can be modified according to the variations of seasonal and temporal conditions of the climatic environment. Therefore it is not possible to verify systematically the changes in the colonization process. Physical measurements thus lead to an approximate estimate of the thickness and coverage percentage of marine bio-fouling. On the other hand, measurements of several platforms from the same site but with different ages can wrap those uncertainties.

The uncertainty in the wave regime is involved in the consideration of the hydraulic parameters, numbers of Reynolds $\left(R_{e}\right)$ and Keulegan-Carpenter $\left(K_{C}\right)$ and characterizing the wave regime. Sources of uncertainty in predicting maxima forces are found at: prediction of the extreme wave and its characteristics (height, wave period and surface profile), the kinematics of water particles near the structure. These uncertainties are reflected in the coefficient of forces $\left(C_{D}\right.$ and $C_{M}$ ) introduced in Morison equation.

Some factors such as orientation of the cylinder, the cylinder condition, the colonization pattern and the wave regime have a significant influence on the hydrodynamic coefficients used in the Morison equation. Laboratory measurements cannot transcribe all this complexity and uncertainties of models must be considered. Therefore it is recommended to use a range of experiments performed and then to assign a confidence score to each author [19]. Schoefs [17], on the site of the North-Sea, showed that the drag coefficient has $60 \%$ influence of effort variance in a node located on the surface. This variable was supposed to be independent hydrodynamic conditions, as suggested in the report of ECOR [22]. The study showed that for an average drag coefficient of 1 , the coefficient of variation of nodal efforts ranged from $17 \%$ to $36 \%$, when the coefficients of variation of $C_{D}$ ranges from 5 to 30 . That was particularly marked between $15 \%$ to $30 \%$ for $C O V$ of $C_{D}$ (usual values), the COV of efforts ranged from $22 \%$ to $36 \%$.

When the flow regime is not post-critical (the digitized curve of API regulation cannot be exploited) it should be referred to the bibliography where the results may differ significantly from one author to another. If a regulation such as API should simplify differences, it is important to analyze the impact if a reliability calculation, incorporating uncertainties, is conducted. To exploit the studies of Theophanatos [12], the relationship between $K_{C}$ and $C_{D}$ is obtained using the empirical curves derived from a large database of experimental works of this author on various wave regimes and tubes with different diameters $(200 \mathrm{~mm}, 314 \mathrm{~mm}, 400 \mathrm{~mm})$. It does not go through a standardized approach via the ratios $C_{D} / C_{D S}$. The advantage is the possibility of working with the results of several authors [23].

\section{MODELING OF MARINE GROWTH ROUGHNESS}

The roughness of marine growth is random and the drag coefficient should be determined from the curves of API RP 2A-WSD. In this paper three approaches, respectively models $\mathrm{R} 1, \mathrm{R} 2$ and $\mathrm{R} 3$ are considered:

- R1 model, consists of using engineering value, $\mathrm{C}_{\mathrm{DS}}=1.05$, to normalize the API curves: $C_{D} / C_{D S}=f\left(K_{C}\right.$ or $\left.K_{C} / C_{D S}\right)$. This approach is called API by default. It does not take into account the roughness of marine growth.

- $\quad$ R2 model is exploiting curves of API directly for the cases which $C_{D S}=f(k / D)=c s t e$. For a smooth or rough element, the value of $\mathrm{C}_{\mathrm{DS}}$ is given respectively by 0.6 or 1.2 . In this approach, $\mathrm{k}$ is considered as time independent.

- R3: For R3 model, a set of roughness of "k" presented in Figure 5, is assumed and $\mathrm{C}_{\mathrm{DS}}$ corresponds as $C_{D S}=f(k(t) / D$ ) (Figure 3). This approach allows measuring the influence of roughness on the drag coefficient and thus the drag force and their changes with the time. $\mathrm{k}$ is here time dependent.

Figure 6 presents the diagrams of drag coefficients $\left(C_{D}\right)$ as a multilinear function of Keulegan-Carpenter numbers $\left(\mathrm{K}_{\mathrm{C}}\right)$ obtained from three above explained approaches.

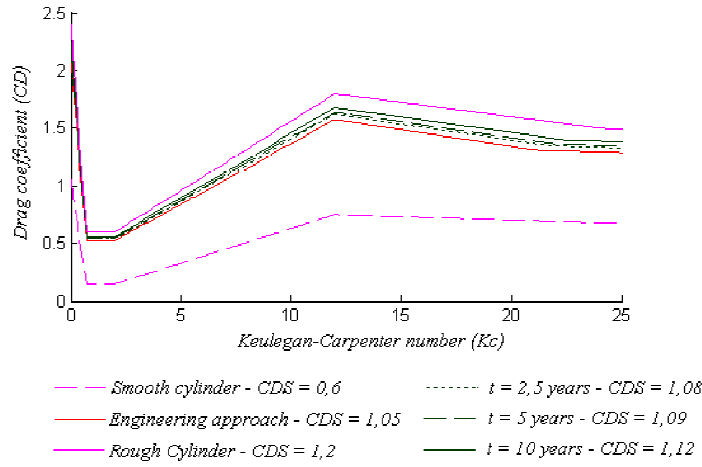

Figure 6. Diagrams of $C D$ in function of $K C$ obtained from $R 1, R 2$ and $R 3$ approaches.

\section{RESULTS}

This study is conducted at Biofouling conditions where three steps are studied: smooth tube (installation or after cleaning), colonized tube after 10 years and colonized tube after 33 years. Morison drag forces at around 10 meters depth are computed by using software developed in the lab and based on the Response Surface modeling of the kinematics field. Monte Carlo simulations allow us to perform a statistical analysis.

The results, presented in Figure 7, demonstrate that between 0 and 10 years, the coefficient of variation of the drag force changes slightly for the three considered approaches, it goes 
from 0.96 to 0.83 . At 33 years, there is a sensitivity in the case of $k$ dependent of $t(C D S=f(k(t) / D)$, the coefficient of variation is less important.

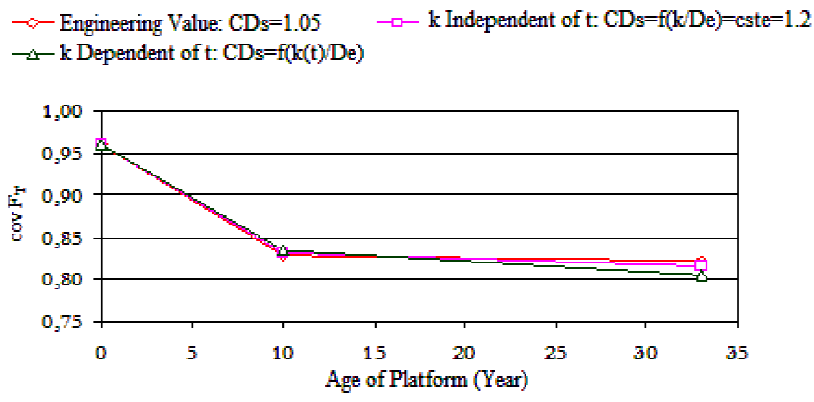

Figure 7. Evolution of cov FT at 0,10 and 33 years.

Figure 8, presents the influence of roughness on the extreme values of drag force, here $90 \%$ percentile, for the three considered approaches, R1, R2 and R3. The results obtained from the case $\mathrm{k}$ independent of $\mathrm{t}(C D S=f(k / D)=c s t e)(\mathrm{R} 3)$, are more conservative than the others for structural analysis. This approach leads to an important increase of drag force.

From 0 to 10 years for both approaches R1 and R3, which use engineering value of $\mathrm{C}_{\mathrm{DS}}$ and $\mathrm{k}$ dependent on it, obtained results are almost similar. After 10 years, there is a difference in the results between the two mentioned approaches. Using engineering value $\mathrm{R} 1$, the results are less conservative for the structural design. However, this approach is less realistic because the effect of roughness in the measuring of hydrodynamic forces is not considered.

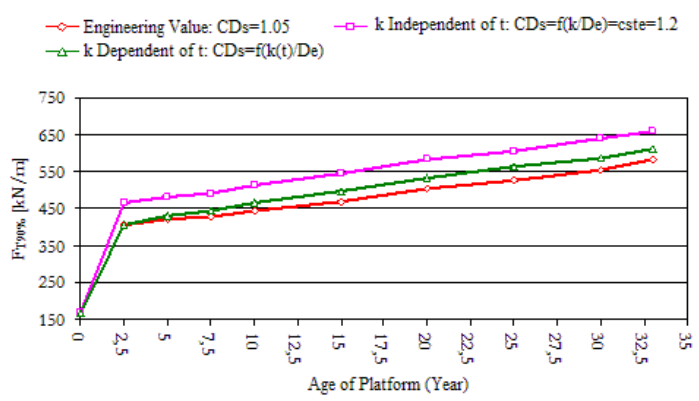

Fig. 8. Evolution of FT90\% versus time and roughness.

\section{CONCLUSIONS}

The marine growth affects the loading of offshore structures due to several factors: overweight, screen effect and modification of hydrodynamic coefficients. This paper focusses on this last point and highlights the important role of the roughness. The paper being devoted to marine growth in the Gulf of Guinea; it suggests a modeling of the roughness of corals with time and quantifies the effect of the probability distribution of forces. It is shown that for these sites, the API principles are no longer fulfilled. The study shows that due to non linear transfers and very fair values for $\mathrm{KC}$, the loading is very sensitive to the modeling of the roughness. A convenient time variant modeling of the roughness can lead to a better representation of around 5\% of the extreme values after 20 years. Authors suggest to investigate properly this key step of the modeling. An alternative study based on the experimental data of artificial macro-roughness will now be carried out to determine more suitable hydrodynamic coefficients for sites the Gulf of Guinea.

\section{REFERENCES}

[1] Wolfram, J., Jusoh, I., Sell, D., 1993, "Uncertainty in the estimation of the fluid loading due to the effects of marine growth", Proc. of 12th int. conf. on Offshore Mechanics and Arctic Engineering, (O.M.A.E’93), Glasgow, vol. II, pp. 219-228.

[2] Compère, C., Segonzac, M., 2002, "Marine growths on offshore structures", Internal report (in French), n ${ }^{\circ}$ R.INT.DITI/GO/MM/99-16 revised, pp. 67.

[3] Schoefs, F., Boukinda, M., Quiniou, V., Birades, M., 2008, "Probabilistic Modeling of Environmental Loading on Jackets in Presence of Marine Growth: Specifities of the Gulf of Guinea", Proc. of 27th International Offshore Mechanics and Arctic Engineering Conference, OMAE2008-57426.

[4] Kirkwood, D., 1981, "Marine fouling and corrosion", Marine Fouling and of Offshore Structures, Society for Underwater Technology, London.

[5] Rodenbusch, G., and Gutierrez, C.A., 1983, "Forces on cylinders in two-dimensional flow", technical report. Vol. 1, BRC 123-83, Bellaire Research Center, Houston, Texas USA.

[6] Boukinda, M., 2007, "Surface de réponse des efforts de houle des structures jackets colonisées par des biosalissures", Thèse de Doctorat, Université de Nantes.

[7] Sarpkaya, T., 1990, "On the Effect of Roughness on Cylinders", Journal of Offshore Mechanics and Artic Engineering, Transactions of the American Society of Mechanical Engineers, Vol.112, pp. 334-340.

[8] Sarpkaya, T., 1976, "In-line and transverse forces on smooth and sand roughened circular cylinders in oscillating flow at high Reynolds numbers", Technical Report $n^{\circ}$ NPS-69SL76062, Naval Post Graduate School, Monterey, CaliforniaUSA.

[9] Sarpkaya, T., 1977, "In-line and transverse forces on cylinders on cylinders in oscillatory flow at high Reynolds numbers”, Journal of Ship Research, Vol.21, N 4 , pp.200216.

[10] Kasahara, Y., Koterayama, W., and Shimazaki, K., 1987, "Wave forces acting on rough circular cylinders at high Reynolds numbers", OTC 5372, Houston, Texas-USA, April 27-30.

[11] Justesen, P., 1989, "Hydrodynamic forces on large cylinders in oscillatory flow", ASCE Journal of Waterway, Portg, Coastal and Ocean Engineering. Vol. 115, N4 July pp. 497-514, vol. 189, pp. 149 - 154. 
[12] Theophanatos, A., 1988, "Marine Growth and Hydrodynamic Loading of Offshore Structures", $\mathrm{PhD}$ thesis, University of Strathclyde, UK.

[13] Morison, J.R., O'Brien, M.P., Johnson, J.W., Schaff, S.A., 1950, "The Force Exerted by Surfaces Waves on Piles", Journal of Petroleum Technology, AIME, Vol. 189.

[14] Shi, W., Park, H.C., Baek, J.H., Kim, Y.C., Shin, H.K., 2012, "Study on the Marine Growth Effect on the Dynamic Response of Offshore Wind Turbines", INTERNATIONAL JOURNAL OF PRECISION ENGINEERING AND MANUFACTURING Vol. 13, No. 7, pp. 1167-1176.

[15] Molin, B., 2002, "Hydrodynamique des Structures Offshore", Guides Pratiques sur les ouvrages en mer, CLAROM, Edition Technip.

[16] Eashwar, M., 1995, "The interrelation of cathodic protection and marine macro fouling", Offshore platform marine electrochemistry cent., pp. 303-312.

[17] Schoefs, F., 2008, "Sensitivity approach for modeling the environmental loading of marine structures through a matrix response surface", Reliability Engineering and System Safety, Available online 19 June 2007, Vol. 93, Issue 7, July 2008, pp. 1004-1017.

[18] Schoefs, F., Boukinda, M., 2010, "Sensitiveness approach for modeling Stochastic Field of Keulegan Carpenter and Reynolds number through a matrix response surface", Journal of Offshore Mechanics and Arctic Engineering, Vol. 132, Iss.1, February 2010, paper $N^{\circ} 011602$ pp. 1-7.

[19] Boukinda, M., Schoefs, F., Quiniou, V., Birades, M., 2007, "Marine Growth Colonization Process in Guinea Gulf: data analysis", Journal of Offshore Mechanics and Arctic Engineering, Vol. 129, Issue 2, pp. 97-106 doi:10.1115/1.2355518.

[20] Schoefs, F., Boukinda, M., 2004, "Modeling of Marine Growth Effect on Offshore Structures Loading Using Kinematics Field of Water Particle", Proc. of 14th International Offshore and Polar Engineering Conference, ISOPE'04, Toulon, Vol. 1, pp. 419-426.

[21] American Petroleum Institute, Recommended Practice for planning, Designing and Constructing Fixed Offshore Platforms Working Stress Design, RP2A-WSD, 21th Edition, 2004.

[22] Engineering Committee on Oceanic Resources, 1991. "Reliability analysis for offshore structures", pp.81.

[23] Sharma, J.N., 1983, "Marine growth on the Hondo platform in the Santa Barbara Channel", 15th Annual Offshore Technology Conference, vol. 2, Dallas U.S.A., pp. 469 - 474. 